\title{
Hospitals' and Nurses’ Preparedness to COVID-19 Pandemic
}

\author{
Rana AbdelFattah Al Awamleh
}

Assistant Professor, Critical Care Nursing, Faculty of Nursing, Jerash University, Jerash, Jordan

\begin{abstract}
Background: Human corona virus disease (COVID-19) is a contagious disease that has been suggested to have a negative impact on both health care system and health care providers, and this was mainly attributed to the rapid and unexpected spread of this pandemic.

Purpose: This study was conducted to assess the level of preparedness of Jordanian nurses and hospitals to COVID-19 pandemic.

Method: cross-sectional, descriptive design was used. Data were collected through electronic survey questionnaires from 306 nurses from seven Jordanian hospitals. A modified version of tool developed by Elhadi and colleagues (2020) was used to collect data regarding the preparedness of nurses and hospitals to COVID19 pandemic.

Results: The majority of nurses (84.3\%) have participated in care provision for clients with COVID-19. Findings of this study revealed that male, older, more experienced nurses scored significantly higher on nurses' preparedness scale. About $60 \%$ of nurse participants perceived their hospitals to be well-prepared to COVID-19 pandemic.

Conclusion: The level of preparedness among nurse was found to be good, but not optimal. Greater emphasis should be placed on younger and less experienced nurses. Urgent actions should be taken to enhance the health institutes' preparedness, particularly in terms of infection control domain.
\end{abstract}

Keywords: COVID-19, Preparedness, Nurses, Hospitals, Pandemic.

\section{INTRODUCTION}

Human corona virus disease (COVID-19) is a contagious disease that is firstly identified in China in 2019, and quickly became a worldwide pandemic. It has been suggested that COVID-19 has a negative impact on both health care system and health care providers, and this was mainly attributed to the rapid and unexpected spread of this pandemic [1]. Increased the burden on the health care system as a result of the COVID-19 was one of the contributing factors to physical and psychological problems among nurses who participated in provision of care to clients with COVID-19 ${ }^{\text {[2] }}$. In order to mitigate the negative impact of COVID-19, various and numerous measures were taken by the vast majority of world's nations to improve the preparedness of their health care institutes. However, many previous studies revealed that the preparedness of health care settings was not up to the optimal level for confronting COVID-19 pandemic. Inadequacy in such preparedness was represented mainly in scarcity of the equipment and medications ${ }^{[3-5]}$, inadequate staffing, and improper hospitals' infrastructure ${ }^{[5-8]}$

Another important factor in determining the preparedness of health care systems was nurses' knowledge about COVID-19 case definition and treatment. Unfortunately, many prior studies showed that knowledge deficit, reliance on social media to get information about COVID-19, and inadequate scientific training programs on COVID-19 management, contributed considerably to improper care of clients with COVID-19 disease ${ }^{[3-6,9-11]}$. Inadequate knowledge about COVID-19 was suggested to be a significant contributor to exaggerated feeling of fear, anxiety, 
psychological distresses, in addition to low self-confidence and low self-esteem, and therefore complicating the mitigation efforts of COVID-19 aftermath ${ }^{[12]}$.

The first confirmed case of COVID19 in Jordan was reported on March 2, 2020. By September 28, 2021, Jordan had 821840 cumulative positive cases and 10703 cumulative deaths. Furthermore, Jordan also reported 798740 expected recoveries [13]. From the first moment of corona virus spread in Jordan, the government had adopted a number of measures to fight COVID-19 pandemic including: establishment of a national control center for COVID-19 crisis, issuing defense laws associated with restricting the movement of citizens, enforcing them to put on masks, and complying with social distancing. Furthermore, Jordanian government implemented partial and full lockdowns over different periods according to the epidemiological status ${ }^{[4,14]}$. However, the Jordanian national reports revealed that inadequate preparedness of healthcare facilities and health care providers, particularly nurses [15]. Accordingly, and due to scarcity of research works addressing the preparedness of Jordanian nurses to COVID-19 pandemic, the current study was conducted to assess the level of preparedness of Jordanian nurses and hospitals to COVID-19 pandemic.

Determining the level of nurses' and hospitals' preparedness might assist in addressing the shortcomings of existing emergency and disaster plans, building effective continuing educational training programs for nurses and other health care providers, and incorporating disaster management in nursing curricula in different Jordanian nursing educational programs.

\section{MATERIALS AND METHODS Study Design}

The researchers used a crosssectional, descriptive comparative design.

Study Participants
Study participants were Jordanian nurses who were working in governmental and private hospitals. Inclusion criteria were Jordanian nurses who had at least one year of clinical experience in governmental and private hospitals. Exclusion criteria were nurses who were working in position where they do not engage directly in care provision of clients, such as administrative positions, at the time of data collection. Using convenience sampling approach, the researcher selected seven Jordanian hospitals (four governmental and three private) to recruit the participants. The sample size was estimated using $\mathrm{G}^{*}$ Power (version 3.1.9.4). The minimum sample size required for this study is 210 , assuming a significance level of 0.05 , an effect size of 0.50 , and a power of 0.95 . To overcome potential participation refusal rate, the researcher sent 450 questionnaires via social media applications to eligible nurse clinicians. The response rate was 68\% (306 nurses).

\section{Instruments}

The questionnaire which was distributed to the participants included two parts: the first part was developed by the researcher and included age, gender, educational level, years of experience, hospital type, working department, and whether they engaged directly in care provision of clients with COVID-19 or not. The second part of questionnaire was the modified version of tool developed by Elhadi and colleagues (2020) to assess the preparedness of nurses and hospitals to COVID-19 pandemic ${ }^{[12]}$. This tool consists of six questions that are related to nurses' perceptions about the proper use of personal protective equipment, participation in COVID-19 management training programs, awareness about procedures of reporting cases and isolation procedures. Furthermore, the tool consists of thirteen questions that focused on nurses' perceptions about the hospitals' preparedness in terms of the availability of PPE, isolation rooms, triage protocol, preparedness plans, protocols of 
reporting (COVID-19) cases among hospitalized patients and healthcare workers, and finally the bed capacity.

The approval from the author has been obtained to use the tool. To check the quality, adequacy, and appropriateness of the complete questionnaire, the researcher conducted a pilot study with a sample of 35 nurses. The respondents revealed that the questionnaire was easy-to-read, easy-tocomprehend and needed an average of 17 minutes to be answered. Reliability analysis of the tool was done which showed Cronbach alpha of 0.79 , which indicated that the tool has a good internal consistency.

\section{Data collection procedure and ethical considerations}

The researchers prepared an electronic questionnaire form through specialized Internet website (Google Forms tool), which enabled the participants to answer the questionnaires anytime and anywhere they want during the period of data collection. The prepared electronic questionnaire was embedded in an electronic attachment link. Each electronic questionnaire started with the consent form that includes the description and purpose of the study, participants' rights to participate, refuse, and withdraw from the study, and confidentiality pledge. After reviewing the consent form, each participant is asked to click "Next" to start answering the questionnaire. After obtaining the approval of the Institutional Review Boards (IRBs) from Jerash University and the targeted hospitals, the researchers contacted the nurse administrator in each participating hospital to assist in identifying potential participants and getting their electronic means of communication to send them the questionnaire electronic link. Data collection procedure was conducted between June and July 2021.

\section{Data analysis}

The data were analyzed using SPSS Statistics 25.0 (IBM Corp., Armonk, N.Y., USA). Means, standard deviations (SD), percentages, and frequencies were computed to describe nurses' characteristics, and nurses' perceptions of their preparedness and hospitals' preparedness. Two-tailed independent t-test and ANOVA test were used to detect if there were any difference in nurses' perceptions based on participants' characteristics and experiences.

\section{RESULTS \\ Participants' characteristics and experiences}

A total of 306 Jordanian nurses participated in this study. The majority of participants were female (64.1\%). Two hundred twenty-one participants (72.2\%) were holding a bachelor's degree in nursing. About $83 \%$ of the participants had a work experience of more than 5 years. About 70\% of the participants were working in governmental hospitals. The majority of nurses (84.3\%) have participated in care provision for clients with COVID-19 cases. Other results related to sociodemographic characteristics were shown in table 1.

\section{Nurses' preparedness to COVID-19 pandemic}

The results of this study revealed that about three-fourths of nurse participants perceived themselves to be well-prepared to COVID-19 pandemic. Keeping up-to-date with COVID-19 case definitions, knowing proper interventions in case of COVID-19 symptoms appearance, and knowing the proper personal protective equipment were found to be the highest-rated nurses' preparedness areas with percentages of $84 \%, 81.7 \%$, and $79.7 \%$ respectively. See table 2 .

Findings of this study revealed that male nurses scored significantly higher on nurses' preparedness scale than female nurses. Regarding age and years of experience, this study showed that older and more experienced nurses had significantly higher scores on preparedness scale than younger and less experienced nurses. Furthermore, scores on nurses' preparedness scale were significantly differed based on 
educational level, in which the participants who were holding bachelor's degree were more prepared to COVID-19 pandemic than those who were holding diploma.
Additionally, nurses who had participated in caring COVID-19 clients showed high level of preparedness rather than who had not (Table 1).

\section{Hospitals' preparedness to COVID-19 pandemic}

Table 1: $t$ test and ANOVA results comparing nurses' preparedness scores based on demographic characteristics and dealing with COVID19 clients $(n=306)$

\begin{tabular}{|c|c|c|c|c|c|}
\hline Characteristic & $\mathbf{N}(\%)$ & $\mathbf{F} / \mathbf{t}$ & $\boldsymbol{p}$ & Mean & SD \\
\hline Gender & & $\mathbf{3 . 2}$ & $\mathbf{0 . 0 0 2}$ & & \\
\hline Female & $196(64.1)$ & & & 10.31 & 1.46 \\
\hline Male & $110(35.9)$ & & & 10.84 & 1.25 \\
\hline Age & & $\mathbf{1 0 . 2 2}$ & $\mathbf{0 . 0 0 5 *}$ & & \\
\hline $18-30$ years & $142(46.4)$ & & & 10.30 & 1.56 \\
\hline $31-40$ years & $127(41.5)$ & & & 10.53 & 1.23 \\
\hline$>40$ years & $37(12.1)$ & & & 11.14 & 1.23 \\
\hline Educational level & & $\mathbf{6 . 0 0}$ & $\mathbf{0 . 0 0 3 * *}$ & & \\
\hline Diploma & $43(14.1)$ & & & 9.93 & 1.70 \\
\hline Bachelor & $221(72.2)$ & & & 10.66 & 1.33 \\
\hline Master/ Doctorate & $42(13.7)$ & & & 10.21 & 1.32 \\
\hline Years of experience & & $\mathbf{8 . 5 6}$ & $<.001 * * *$ & & \\
\hline 1 - 5 years & $141(46.1)$ & & & 10.26 & 1.59 \\
\hline 6 - 10 years & $54(17.6)$ & & & 10.22 & 1.25 \\
\hline$>10$ years & $111(36.3)$ & & & 10.93 & 1.12 \\
\hline Type of hospital & & $\mathbf{0 . 5 4 2}$ & $\mathbf{0 . 5 8 8}$ & & \\
\hline Governmental & $211(69)$ & & & 10.53 & 1.36 \\
\hline Private & $95(31)$ & & & 10.43 & 1.51 \\
\hline Work department & & $\mathbf{0 . 4 3 3}$ & $\mathbf{0 . 8 5 7}$ & & \\
\hline Emergency & 65 & & & 10.58 & 1.488 \\
\hline Medical-Surgical & 105 & & & 10.46 & 1.366 \\
\hline Maternity and labour & 24 & & & 10.67 & 1.373 \\
\hline Paediatric & 32 & & & 10.38 & 1.431 \\
\hline Outpatient & 11 & & & 10.18 & 1.779 \\
\hline Haemodialysis & 4 & & & 11.25 & 1.500 \\
\hline Critical Care Unit & 65 & & & 10.48 & 1.371 \\
\hline Dealing with COVID-19 clients & & $\mathbf{2 . 1 1 2}$ & $\mathbf{0 . 0 3 5}$ & & \\
\hline Yes & $258(84.3)$ & & & 10.57 & 1.36 \\
\hline No & $48(15.7)$ & & & 10.10 & 1.61 \\
\hline \multicolumn{2}{|l|}{} & & & \\
\hline
\end{tabular}

Table 2: Nurses' preparedness to COVID-19 pandemic

\begin{tabular}{|l|l|l|}
\hline \multicolumn{1}{|c|}{ Items } & \multicolumn{1}{|c|}{ Yes n (\%) } & No n (\%) \\
\hline Are you following the latest updates regarding case definitions for COVID19? & $257(84)$ & $49(16)$ \\
\hline Did you receive training courses in COVID-19 outbreak management? & $191(62.4)$ & $115(37.6)$ \\
\hline Do you know the proper personal protective equipment (PPE)? & $244(79.7)$ & $62(20.3)$ \\
\hline Are you able to implement isolation procedures for COVID-19 effectively? & $209(68.3)$ & $97(31.7)$ \\
\hline Do you know the proper protocol of reporting of active or suspected COVID-19 cases & $217(70.9)$ & $89(29.1)$ \\
\hline Do you know the proper protocol if symptoms of COVID-19 appear on you & $250(81.7)$ & $56(18.3)$ \\
\hline
\end{tabular}

Table 3: Hospitals' preparedness to COVID-19 pandemic

\begin{tabular}{|l|c|c|}
\hline \multicolumn{1}{|c|}{ Items } & Yes n (\%) & No n (\%) \\
\hline Do you consider your hospital well prepared to fight the COVID19 pandemic? & $182(59.5)$ & $124(40.5)$ \\
\hline Is your hospital equipped with a negative-pressure Airborne Infection Isolation Rooms? & $92(30.1)$ & $214(69.9)$ \\
\hline Does your hospital provide proper personal protective equipment (PPE)? & $137(44.8)$ & $169(55.2)$ \\
\hline Does your hospital follow a specific protocol or triage for isolation of patients with symptoms of COVID19? & $151(49.3)$ & $155(50.7)$ \\
\hline $\begin{array}{l}\text { A designated location, separate from other clinical triage and evaluation areas, (utilizing the principles of } \\
\text { social distancing) for the admission of patients with possible COVID-19 has been determined }\end{array}$ & $155(50.7)$ & $151(49.3)$ \\
\hline Availability of face masks at the hospital entrance & $188(61.4)$ & $118(38.6)$ \\
\hline Availability of alcohol-hand sanitizer dispenser in all units and corridors of hospital & $182(59.5)$ & $124(40.5)$ \\
\hline A copy of the hospital COVID-19 preparedness plan is available at the facility and accessible by staff & $133(43.4)$ & $173(56.6)$ \\
\hline When scheduling appointments, special instructions being provided to patients by your institution. & $153(50)$ & $153(50)$ \\
\hline $\begin{array}{l}\text { A written protocol has been developed for identifying, monitoring and reporting COVID-19 among } \\
\text { hospitalized patients, volunteers, and staff (e.g., weekly or daily number of patients and staff with COVID-19) }\end{array}$ & $129(42.2)$ & $177(57.8)$ \\
\hline Surge capacity plans include strategies to help increase hospital bed capacity and number of staff available & $145(47.4)$ & $161(52.6)$ \\
\hline Criteria have been developed for determining when to cancel elective admissions and surgeries & $121(39.5)$ & $185(60.5)$ \\
\hline A procedure has been developed for communicating changes in hospital status to health authorities & $185(60.5)$ & $121(39.5)$ \\
\hline
\end{tabular}


The results of this study revealed that about $60 \%$ of nurse participants perceived their hospitals to be well-prepared to COVID-19 pandemic. Availability of facemasks, presence of procedure for communicating changes in hospital status to health authorities, and availability of handsensitizers were found to be the highestrated hospitals' preparedness areas with percentages of $61.4 \%, 60.5 \%$, and $59.5 \%$ respectively. See table 3.

\section{DISCUSSION}

Contradictory to many previous studies [12, 16-17], the findings of the current study revealed that the majority of nurse participants expressed high level of preparedness regarding COVID-19 pandemic. This study was conducted fifteen months after emergence of first case of COVID-19 in Jordan. Through that time, the Jordanian Ministry of Health has been following up on the latest updates issued by world health organization (WHO) on COVID-19 case definitions, prevention, and management. Furthermore, Ministry of Health provides reliable information about Covid-19 to the public and health care providers on an ongoing basis ${ }^{[4]}$. This might explain why the vast majority of nurse participants were following the latest updates regarding case definitions for COVID19.

The findings of this study demonstrated that the great majority of nurse participants have mastered the use of personal protective equipment (PPE). This is congruent with the work of Alzyoud and colleagues (2021) who found that despite proficiency of nurses in wearing masks, they still have knowledge deficit about COVID-19 transmission modes and prevention protocols ${ }^{[18]}$. This might be attributed to inadequate involvement in training workshops regarding to COVID-19 transmission and associated infection control measures, and this was apparent in our findings, as that about nearly $40 \%$ of nurse participants did not receive any training courses addressing COVID-19.
Regarding demographic characteristics, our study found that male nurse participants were perceived to be more prepared to COVID-19 than female nurses were. This is incongruent with previous study conducted by Chanie and colleagues (2021) who revealed that male and unmarried health care providers had low levels of preparedness to COVID-19 [16]. However, our findings were in line with a study conducted by Al-Ashwal (2020) who attributed high level of preparedness of male health care providers to the tendency of females to underestimate their proficiency and mastery of skills ${ }^{[19]}$.

Older and more experienced nurse participants were found to have higher level of preparedness to COVID-19. This is consistent with findings of Chua, Cosmas, and Arsat (2021), and it might be explained as that experienced nurses might have benefited from their experience in dealing with previous epidemics or pandemics, either at local or international levels [17]. And this might explain the higher level of preparedness that was reported by nurse participants who have participated in care provision for clients with COVID-19 in our study in comparison to those who have not.

About $60 \%$ of nurse participants stated that their hospitals were wellprepared to fight COVID-19. Availability of a negative-pressure airborne infection isolation rooms was perceived by study participants as the most lacking items of hospitals' preparedness items. This was consistent with findings of $\mathrm{Al}$ Baalharith and Pappiya (2021) study. Although availability of facemasks and hand sanitizers were perceived as the most satisfactory items of hospitals' preparedness, they were not yet at the optimum level ${ }^{[20]}$.

\section{CONCLUSION}

The level of preparedness among nurses was found to be good, but not optimal. Greater emphasis should be placed on younger and less experienced nurses. Urgent actions should be taken to enhance 
the health institutes' preparedness, particularly in terms of infection control domain.

\section{Limitations of the Study}

The main limitation of this study was the use of convenience sampling technique. This implies that the results presented might not be truly representative to all nurses who were working in Jordanian governmental, private, and military hospitals. Future studies should include a sample of nurses from other hospitals, so that the results can be more generalizable.

\section{Acknowledgement: None}

Conflict of Interest: The authors declare that they have no known competing financial interests or personal relationships that could have appeared to influence the work reported in this paper.

Source of Funding: This research received no specific grant from any funding agency or commercial sectors.

\section{Ethical Approval: Approved}

\section{REFERENCES}

1. Urdaneta F, Stacey M, Sorbello M. The Adverse Impact of COVID-19 on Health Care Providers: Time to Start Measuring. Anesthesia \& Analgesia. 2020;131. doi:10.1213/ane.0000000000005088

2. Shreffler J, Huecker M, Petrey J. The Impact of COVID-19 on Healthcare Worker Wellness: A Scoping Review. West J Emerg Med. 2020;21. doi:10.5811/westjem.2020.7.48684

3. Suleiman. A, Bsisu. I, Guzu. H , Santarisi. A , Alsatari. M , Abbad. A , Jaber. A, et al. (2020). Preparedness of Frontline Doctors in Jordan Healthcare Facilities to COVID-19 Outbreak. Int J Environ Res Public Health, 17 (3181), pp. 1-11

4. Alqutob $\mathrm{R}, \mathrm{Al}$ Nsour $\mathrm{M}$, Tarawneh MR, Ajlouni M, Khader Y, Aqel I, Kharabsheh S, Obeidat N. (2020). COVID-19 Crisis in Jordan: Response, Scenarios, Strategies, and Recommendations. JMIR Public Health Surveill. 2020 Jul 7;6(3):e19332. doi:
10.2196/19332. PMID: 32407289; PMCID: PMC7381018

5. Shrestha. G.S, Paneru. H.R, Acharya. S.P, Shrestha. S.K, Sigdel.M.R, Tiwari. S, Yadav.B.K, Rijal.B, et al. (2020). Preparedness for Coronavirus Disease in Hospitals of Nepal: A Nationwide Survey. JNMA, 58 (224), pp. 248-251.

6. Al-Ashwal FY, Kubas M, Zawiah M, Bitar AN, Mukred Saeed R, Sulaiman SAS, et al. (2020) Healthcare workers' knowledge, preparedness, counselling practices, and perceived barriers to confront COVID-19: A cross-sectional study from a war-torn country, Yemen. PLoS ONE 15(12): e0243962. https://doi.org/10.1371/journal. pone. 0243962 .

7. Baalharith IMA, Pappiya EM. Nurses preparedness and response to COVID-19. Int J Africa Nurs Sci. 2021;14:100302. doi:10.1016/j.ijans.2021.100302

8. Khan MF, Mahmood M, Hasrat MN, Javed B, Javed O. Perception, preparedness and response of health care personals towards COVID-19 pandemic in Azad Jammu \& Kashmir, Pakistan: A cross sectional interview based study. Clin Epidemiology Glob Health. 2021;11:100783. doi:10.1016/ j.cegh.2021.100783

9. Elhence A, Sureka B, Garg MK, Bhardwaj P, Kanchan T, Sinha A, et al. (2020). Preparing for a COVID-19 pandemic: Redefining roles of multidisciplinary team in a large tertiary hospital in Rajasthan. J Family Med Prim Care 2020;9:5439-44

10. Chatterjee, Argha \& Biswas, Bivas \& Gehani, Anisha \& Das, Jayanta \& Sen, Saugata \& Mukhopadhyay, Sumit \& Chandra, Aditi \& Ghosh, Priya \& Gupta, Bharat \& Lingegowda, Dayanandal. Oncoradiology Preparedness in the COVID19 Pandemic: Perspective from a Tertiary Oncology Referral Center from Eastern India. Indian J Med Paediatr Oncol. 2020, 41. 10.4103/ijmpo.ijmpo_240_20.

11. Desalegn Z, Deyessa N, Teka B, Shiferaw W, Yohannes $M$, Hailemariam D, et al. (2021) Evaluation of COVID-19 related knowledge and preparedness in health professionals at selected health facilities in a resource-limited setting in Addis Ababa, Ethiopia. PLoS ONE 16(2): e0244050. https://doi.org/10.1371/journal.

pone. 0244050 
12. Elhadi. M, Msherghi. A, Alkeelani. M, Zorgani. A, Zaid. A, Alsuyihili. A, Buzreg. A, et al. (2020). Assessment of Healthcare Workers' Levels of Preparedness and Awareness Regarding COVID-19 Infection in Low-Resource Settings. Am. J. Trop. Med. Hyg., 103(2), 2020, pp. 828-833. doi:10.4269/ajtmh.20-0330

13. Jordan Ministry of Health. 2021. Retrieved from:

https://app.powerbi.com/view?r=eyJrIjoiZW ZlOTAxOGItMmY3ZS00MzMxLWE3Mm ItZWU4ZGViMTlkNTUwIiwidCI6IjM3MjI 3YTljLWI1OGUtNGNiNi05NDNhLWI2Zj E5ZmJjZWFjMCIsImMiOj19

14. Al-Tammemi AB. The Battle Against COVID-19 in Jordan: An Early Overview of the Jordanian Experience. Front. public health. 2020;8. doi:10.3389/fpubh.2020. 00188.

15. National Center for Security and Crises Management. COVID-19 in Jordan. (2020). Available online at: http://ncscm.gov.jo/index.php (accessed April 16, 2020)

16. Chanie ES, Feleke DG, Fetene S, Tigabu A, Asnakew S, Tiruneh $\mathrm{T}$, et al. Level of Preparedness for COVID-19 and Its Associated Factors among Frontline Healthcare Providers in South Gondar Public Hospitals, Northwest Ethiopia, 2020: A Multicenter Cross-Sectional Study.
BioMed Research International 2021;2021: 1-8. doi:10.1155/2021/6627430

17. Chua BS, Cosmas G, Arsat N. Nurses' Preparedness, Readiness, and Anxiety in Managing COVID-19 Pandemic. Asia Pac J Public Health. 2021;33:564-70. doi:10.1177/10105395211012170

18. Alzyoud, et al. Nurses Knowledge Regarding Prevention Protocol of COVID19 in Emergency Departments. MedicoLegal Update 2021. doi:10.37506/mlu.v21i3.2979

19. Al-Ashwal FY, Kubas M, Zawiah M, Bitar AN, Saeed RM, Sulaiman SAS, et al. Healthcare workers' knowledge, preparedness, counselling practices, and perceived barriers to confront COVID-19: A cross-sectional study from a war-torn country, Yemen. Plos One 2020;15. doi:10.1371/journal.pone.0243962

20. Baalharith IMA, Pappiya EM. Nurses preparedness and response to COVID-19. Int J Africa Nurs Sci. 2021;14:100302. doi:10.1016/j.ijans.2021.100302.

How to cite this article: Rana AbdelFattah Al Awamleh. Hospitals' and nurses' preparedness to COVID-19 pandemic. International Journal of Science \& Healthcare Research. 2021; 6(4): 159-165. DOI: https://doi.org/10.52403/ijshr. 20211022 